\title{
Properness criteria for families of coherent analytic sheaves
}

\author{
Matei Toma
}

\begin{abstract}
We extend Langton's valuative criterion for families of coherent algebraic sheaves to a complex analytic set-up. As a consequence, we derive a set of sufficient conditions for the compactness of moduli spaces of semistable sheaves over compact complex manifolds. This also applies to some cases appearing in complex projective geometry not covered by previous results.
\end{abstract}

\section{Introduction}

There is a variety of situations when moduli spaces of semistable coherent sheaves over projective schemes are known to exist; see [HL10] for an extensive treatment of this topic. Such moduli spaces typically turn out to be projective. This is often proved by using a result of Langton, who checked that Grothendieck's valuative criterion for properness applies to families of semistable sheaves [Lan75], [HL10, Theorem 2.B.1]. Sometimes even if the base variety $X$ is projective, in order to define stability, one may be led to consider arbitrary real ample classes as polarizations; cf. [GT17, GRT16b, GRT16a, CP19]. However, when the polarization is irrational, Langton's result does not directly apply, and its proof cannot be adapted in a straightforward way to such a case.

When the base variety is compact complex analytic, one may still speak of semistability with respect to Gauduchon metrics. But even over compact Kähler manifolds, a result analogous to Langton's is not available, and complex analytic moduli spaces for semistable sheaves have been constructed only in special cases.

It is the purpose of this paper to provide replacements of properness valuative criteria as in Langton's result in a complex analytic set-up and to show how they may be applied to prove the compactness of moduli spaces of semistable sheaves. For coherent sheaves over a compact complex manifold, a fairly general semistability notion will be described in Section 2. (We define semistability only for pure sheaves, but we later give analogues of our statements in the non-pure case.) Then in Section 3, we deal with families of semistable sheaves over one-dimensional bases. In this context, we prove Theorem 3.1, an analogue of which over spectra of discrete valuation rings already provides a solution to the case of irrational polarizations mentioned above. This theorem basically says that for a flat family of coherent sheaves with general semistable members over a curve, one may replace the special members in such a way that the resulting family has

Received 19 January 2018, accepted in final form 8 August 2019.

2010 Mathematics Subject Classification 32G13, 14D20.

Keywords: semistable coherent sheaves, moduli of sheaves, properness criteria.

This journal is (C) Foundation Compositio Mathematica 2020. This article is distributed with Open Access under the terms of the Creative Commons Attribution Non-Commercial License, which permits non-commercial reuse, distribution, and reproduction in any medium, provided that the original work is properly cited. For commercial re-use, please contact the Foundation Compositio Mathematica. 


\section{FAMILIES OF COHERENT ANALYTIC SHEAVES}

only semistable fibres. When the base manifold is not projective, however, such a result on onedimensional families may no longer be sufficient. This is related to the fact that the two current definitions of meromorphic mappings do not coincide. Stoll's definition, which uses extensions along curves, is weaker than Remmert's, which requires a factorization through a proper modification [Sto58, text following Satz 8.7, pp. 424-428]; see also [Hir80]. In Section 4, we prove Theorem 4.1, which provides a replacement for Langton's valuative criterion that also holds for families over higher-dimensional bases, albeit under a more restrictive semistability condition. Using this result, in Section 5 we give sufficient conditions for a moduli space of semistable sheaves over a compact complex manifold to be compact. The paper ends with a criterion for separatedness (Theorem 6.4).

For Theorem 3.1, we follow Langton's original line of proof with two new inputs, one of combinatorial nature which compensates the lack of rationality of the polarizations involved, and one application of Artin approximation which avoids non-properness issues of the relative Douady space. Theorems 4.1 and 5.1 are essentially new, and they are especially pertinent to the complex analytic context. Their proofs depend on a notion of boundedness for sets of isomorphy classes of coherent analytic sheaves which was introduced in [Tom16].

\section{Degree functions and stability}

Various definitions of semistability for coherent sheaves on projective manifolds are in use, and many recent papers aim at a formalization of their properties; see, for example, [Joy07, And09].

Here we content ourselves with the presentation of the stability notion which will appear in the results of this paper. It will use a generalization of the Hilbert polynomial of a coherent sheaf which will make sense in a complex geometric (not necessarily projective) framework. This notion finds applications even when the base space $X$ is a smooth projective variety; cf. [GT17, GRT16b, GT20]. In order to introduce it, one may choose to work either on a category $\operatorname{Coh}_{d}(X)$ of coherent sheaves of dimension at most $d$ on an analytic space $X$ or on a quotient category $\operatorname{Coh}_{d, d^{\prime}}(X):=\operatorname{Coh}_{d}(X) / \operatorname{Coh}_{d^{\prime}-1}(X)$ as in $[H L 10, \S 1.6]$. In this paper, we chose the first, but the statements can be easily rephrased to stay valid for the second approach.

From now on, $X$ is a compact analytic space of dimension $n$, and $d$ and $d^{\prime}$ denote integers satisfying $n \geqslant d \geqslant d^{\prime} \geqslant 0$. In particular, $X$ may be the associated analytic space of a proper algebraic space over $\mathbb{C}$. We denote the Grothendieck group of coherent sheaves on $X$ by $K_{0}(X)=$ $K_{0}(\operatorname{Coh}(X))$ and the class in $K_{0}(X)$ of a coherent sheaf $F$ by $[F]$. If $F$ has dimension at most $p$, we write $\operatorname{cycle}_{p}(F)$ for the $p$-cycle associated with $F$.

Definition 2.1 (Degrees). Consider a group morphism $\operatorname{deg}_{p}: K_{0}(X) \rightarrow \mathbb{R}$ inducing a positive map on non-zero $p$-cycles when putting $\operatorname{deg}_{p}(Z):=\operatorname{deg}_{p}\left(\left[\mathcal{O}_{Z}\right]\right)$ for irreducible $p$-cycles $Z$ and the following properties:

(1) The equality $\operatorname{deg}_{p}([F])=\operatorname{deg}_{p}\left(\operatorname{cycle}_{p}(F)\right)$ holds for any $F \in \operatorname{Coh}_{p}(X)$.

(2) If a set of positive $p$-cycles is such that $\operatorname{deg}_{p}$ is bounded on it, then $\operatorname{deg}_{p}$ takes only finitely many values on this set.

(3) The function $\operatorname{deg}_{p}$ is continuous on flat families of sheaves.

(4) The function $\operatorname{deg}_{p}$ is locally constant on flat families of sheaves.

We call such a function a degree function in dimension $p$ if it has properties (1), (2) and (3). If 
only the first property is satisfied, we call it a weak degree function in dimension $p$, and if $\operatorname{deg}_{p}$ has all four properties, we call it a strong degree function in dimension $p$. For simplicity, we write $\operatorname{deg}_{p}(F)=\operatorname{deg}_{p}([F])$ for any $F \in \operatorname{Coh}(X)$.

A collection of degree functions $\left(\operatorname{deg}_{d}, \ldots, \operatorname{deg}_{d^{\prime}}\right)$ in dimensions $d$ to $d^{\prime}$ on $X$ is called a $\left(d, d^{\prime}\right)$ degree system and similarly for weak or strong degree functions.

Note that for any weak degree function $\operatorname{deg}_{p}$ in $\operatorname{dimension} p$ on $X$, one $\operatorname{has} \operatorname{deg}_{p}(F)>0$ if $F$ is $p$-dimensional and $\operatorname{deg}_{p}(F)=0$ if $F \in \operatorname{Coh}_{p-1}(X)$.

Strong degree functions appear naturally if $X$ is endowed with differential forms $\omega_{p}$ of degree $2 p$ which are d-closed and such that their $(p, p)$-components $\omega_{p}^{p, p}$ are strictly positive in Lelong's sense; cf. [BM14, §§III.2.4 and IV.10.6]. In this case, for $F \in \operatorname{Coh}(X)$, one defines $\operatorname{deg}_{p}(F):=\int_{\tau_{p}(F)} \omega_{p}$, where $\tau(F)$ is the homological Todd class of $\mathcal{F}$ and the integral is computed on a semianalytic representative of $\tau_{p}(F)$; cf. [Tom16, §2], [BH69, DP77], [AG06, Theorem 7.22], [Gor81, §8.4], [Her66]. These functions satisfy condition (2) of Definition 2.1 since they are constant on connected components of the corresponding cycle spaces and since no analytic space can have an infinite number of irreducible components accumulating at a point. When $(X, \omega)$ is a Kähler space, cf. [Var89, §II.1.2], we obtain such $2 p$-forms as $p$-powers of the Kähler form $\omega$. When $\left(X, \mathcal{O}_{X}(1)\right)$ is a projective variety endowed with an ample line bundle, by taking $\omega$ to be a strictly positive curvature form of $\mathcal{O}_{X}(1)$, we recover the coefficient of the Hilbert polynomial of $F$ in degree $p$ as $\operatorname{deg}_{p}(F) / p$ !. However, even on projective manifolds, one is naturally led to consider degree functions which are not associated with an ample polarization; cf. [GT17, GRT16b, GRT16a, CP19, GKP16].

In the above situation, the condition $\mathrm{d} \omega_{p}^{p, p}=0$ implies that the corresponding degree function is locally constant on flat families of sheaves. An example having found applications in the literature where only continuity holds is that of degree functions on non-Kählerian compact manifolds. Such a manifold always carries a Gauduchon form $\omega_{n-1}$, that is, a positive $(n-1, n-1)$ form $\omega_{n-1}$ such that $\partial \bar{\partial} \omega_{n-1}=0$. One then defines degree functions in dimensions $n$ and $n-1$ by setting

$$
\operatorname{deg}_{n}(F):=\operatorname{rk}(F) \text { and } \operatorname{deg}_{n-1}(F):=\int_{X}\left[\omega_{n-1}\right]_{A} \cdot c_{1}(F)_{B C},
$$

where the classes are computed in Aeppli cohomology and in Bott-Chern cohomology, respectively; cf. [LT95, Tel10]. More generally, any strictly positive $\partial \bar{\partial}$-closed $(p, p)$-form on $X$ gives rise to a degree function in dimension $p$. One shows that condition (2) of Definition 2.1 is satisfied by the same argument as in the Kähler case. Indeed, such a function is pluriharmonic on the cycle space by [Bar78, Proposition 1], and attains its minimum on any closed subset of the cycle space by Bishop's theorem. It is therefore constant on any irreducible component of this space. Note that any compact complex manifold $X$ admits a degree function in dimension zero defined by $\operatorname{deg}_{0}(F):=\int_{X} \operatorname{ch}_{n}(F)$. In this way, Gauduchon surfaces $\left(X, \omega_{1}\right)$ get a $(2,0)$-degree system.

For the following definition, the order relation we consider on $\mathbb{R}^{d-d^{\prime}+1}$ is the lexicographic order.

Definition 2.2 (Semistability). Suppose that $X$ is equipped with a weak $\left(d, d^{\prime}\right)$-degree system $\left(\operatorname{deg}_{d}, \ldots, \operatorname{deg}_{d^{\prime}}\right)$. For any $d$-dimensional coherent sheaf $F$, we define its slope vector with respect to this system as

$$
\mu(F):=\left(\frac{\operatorname{deg}_{d}(F)}{\operatorname{deg}_{d}(F)}, \frac{\operatorname{deg}_{d-1}(F)}{\operatorname{deg}_{d}(F)}, \ldots, \frac{\operatorname{deg}_{d^{\prime}}(F)}{\operatorname{deg}_{d}(F)}\right) \in \mathbb{R}^{d-d^{\prime}+1} .
$$




\section{FAMILIES OF COHERENT ANALYTIC SHEAVES}

A $d$-dimensional sheaf $F$ is called slope-semistable or just semistable if it is pure and if for any non-trivial subsheaf $E \subset F$, we have $\mu(E) \leqslant \mu(F)$.

Note that in the case $d^{\prime}=d$, semistability on $\operatorname{Coh}_{d}(X)$ just means $d$-dimensional purity.

With literally the same proof as in [HL10, §1.3], one checks the existence of a HarderNarasimhan filtration for the above semistability notion.

Theorem 2.3. With respect to a weak $\left(d, d^{\prime}\right)$-degree system on $X$, any pure $d$-dimensional sheaf $F$ admits a unique increasing filtration

$$
0=H N_{0}(F) \subset H N_{1}(F) \subset \cdots \subset H N_{l}(F)=F
$$

with semistable factors $H N_{i}(F) / H N_{i-1}(F)$ for $1 \leqslant i \leqslant l$ and such that

$$
\mu\left(H N_{1}(F) / H N_{0}(F)\right)>\cdots>\mu\left(H N_{l}(F) / H N_{l-1}(F)\right) .
$$

In particular, under the above hypotheses, $H N_{1}(F)$ has the properties of a maximal destabilizing subsheaf of $F$; that is, for all subsheaves $E \subset F$, one has $\mu(E) \leqslant \mu\left(H N_{1}(F)\right)$, and in case of equality, one has $E \subset H N_{1}(F)$. Moreover, $H N_{1}(F)$ is a saturated subsheaf of $F$; that is, the quotient $F / H N_{1}(F)$ is either zero or pure $d$-dimensional.

Before we go on to the relative case, let us remark that purity is a Zariski-open property in flat families of coherent sheaves. Indeed, if $S$ is any analytic space and if $E$ is a flat family of $d$-dimensional coherent sheaves on the fibres of $X$ parametrized by $S$, then one can adapt Maruyama's approach in [Mar96, Proposition 1.13] to prove that the set of points $s \in S$ such that $E_{s}$ is not pure is a closed analytic subset of $S$. It suffices to work in loc. cit. with local resolutions and apply the purity criterion from [Mar96, Lemma 1.12].

By a relative Harder-Narasimhan filtration for a flat family $E$ of $d$-dimensional coherent sheaves on $X$ parametrized by an irreducible analytic space $S$, we mean a proper bimeromorphic morphism of irreducible analytic spaces $T \rightarrow S$ together with a filtration

$$
0=H N_{0}(E) \subset H N_{1}(E) \subset \cdots \subset H N_{l}(E)=E_{T}
$$

such that the factors $H N_{i}(E) / H N_{i-1}(E)$ are flat over $T$ for $1 \leqslant i \leqslant l$ and which induces the absolute Harder-Narasimhan filtrations fibrewise over some dense Zariski-open subset of $S$, cf. [Tom19] for a more general situation and [HL10, §2.3] for the projective algebraic case.

In order to obtain a relative Harder-Narasimhan filtration for a family of sheaves, we need stronger assumptions on the degree functions. Using the techniques of [Tom16], the following result is obtained in [Tom19].

Theorem 2.4. Suppose that $X$ is endowed with a strong $\left(d, d^{\prime}\right)$-degree system induced by a system of strictly positive $\partial \bar{\partial}$-closed differential forms. Then with respect to the corresponding semistability notion, every flat family $E$ of $d$-dimensional coherent sheaves on $X$ with pure general members parametrized by an irreducible analytic space $S$ has a relative Harder-Narasimhan filtration $\left(T \rightarrow S, H N_{\bullet}(E)\right)$. Moreover, this filtration has the following universal property: if $f: T^{\prime} \rightarrow S$ is a bimeromorphic morphism of irreducible analytic spaces and if $F_{\bullet}$ is a filtration of $E_{T^{\prime}}$ with flat factors which coincides fibrewise with the absolute Harder-Narasimhan filtration over general points $s \in S$, then $f$ factors over $T$ and $F_{\bullet}=H N_{\bullet}(E)_{T^{\prime}}$.

A consequence of this theorem is the fact that semistability is a Zariski-open property in flat families of sheaves. 


\section{TOMA}

Another way to look at the relative Harder-Narasimhan filtration is to consider its direct image over $X \times S$ and the filtration which this induces on $E$. In particular, if $E$ is a family as in the statement of Theorem 2.4 whose general fibres are not semistable and if $\left(f: T \rightarrow S, H N_{\bullet}(E)\right)$ is the relative Harder-Narasimhan filtration of $E$, then the fibres over general points $s \in S$ of the image $F_{1}$ of the composition of sheaf homomorphisms

$$
\left(\operatorname{id}_{X} \times f\right)_{*}\left(H N_{1}(E)\right) \rightarrow\left(\operatorname{id}_{X} \times f\right)_{*}\left(E_{T}\right) \rightarrow E
$$

coincide with $H N_{1}\left(E_{s}\right)$. We call the sheaf $F_{1}$ the relative maximal destabilizing sheaf of $E$.

\section{One-dimensional families}

In this section, we deal with the case of one-dimensional families in its analytic formulation. The attentive reader will be able to translate the argument in terms of families over the spectrum of a discrete valuation ring, when the base space is algebraic, with some care however when applying Artin approximation. We denote the open unit disc in $\mathbb{C}$ by $\mathbb{D}$ and write $\mathbb{D}^{*}:=\mathbb{D} \backslash\{0\}$.

Theorem 3.1. Let $X$ be a compact complex manifold endowed with a $\left(d, d^{\prime}\right)$-degree system, and let $F$ be a $\mathbb{D}$-flat family of $d$-dimensional sheaves on $X$. Suppose that for $s \in \mathbb{D} \backslash\{0\}$, the fibres $F_{s}$ are semistable. Then there exists a coherent subsheaf $F^{\prime} \subset F$ coinciding with $F$ over $\mathbb{D} \backslash\{0\}$ and such that the fibre $F_{0}^{\prime}$ over zero is also semistable.

Before starting the proof, note that $\mathcal{O}_{\mathbb{D}, s}$ is a principal ideal domain for any $s \in \mathbb{D}$, so for an $\mathcal{O}_{\mathbb{D}, s}$-module, being flat boils down to being torsion free. Thus, since $F$ has no $\mathbb{D}$-torsion, any coherent subsheaf of $F$ continues to be flat over $\mathbb{D}$.

Proof. The proof follows the line of $[\mathrm{HL} 10, \S 2 \mathrm{~B}]$ with an essential expansion due to the lack of discreteness of the degree functions in our set-up. A smaller change appears at the end where we avoid the issue on the use of the properness of the relative Douady space and replace it with a short argument based on Artin approximation.

For any integer $\delta \in\left[d^{\prime}, d\right]$, we consider semistability with respect to the $(d, \delta)$-degree system $\left(\operatorname{deg}_{d}, \ldots, \operatorname{deg}_{\delta}\right)$ obtained by restricting the given $\left(d, d^{\prime}\right)$-degree system on $X$. We call a semistable sheaf with respect to such a restricted system $(d, \delta)$-semistable for short. Note that a sheaf is $(d, d)$-semistable if and only if it is pure of dimension $d$. Thus for $d^{\prime}=d$, Theorem 3.1 just says that if $F$ is flat and with pure fibres over $\mathbb{D}^{*}$, then a subsheaf $F^{\prime} \subset F$ exists with $F_{\mathbb{D}^{*}}^{\prime}=F_{\mathbb{D}^{*}}$ and $F_{0}^{\prime}$ pure as well. This is the case $\delta=d$ of the following claim, and a proof is suggested in [HL10, Exercise 2.B.2]. Since this special case works under weaker hypotheses and has an easier proof, we provide a separate statement for it as Proposition 3.3.

Claim 3.2. Let $X$ and $F$ be as in Theorem 3.1 and $d \geqslant \delta \geqslant d^{\prime}$. Then, if $F_{0}$ is moreover $(d, \delta+1)$ semistable, there exists a subsheaf $F^{\prime} \subset F$ with $F_{\mathbb{D}^{*}}^{\prime}=F_{\mathbb{D}^{*}}$ and $(d, \delta)$-semistable fibre $F_{0}^{\prime}$ over zero. (Here when $\delta=d$, we think of the $(d, d+1)$-semistability condition as being void; that is, all sheaves $F_{0}$ are automatically $(d, d+1)$-semistable.)

Theorem 3.1 follows from this claim by descending induction on $\delta$.

We now proceed to the proof of Claim 3.2. The case $\delta=d$ has already been discussed, so we will work under the hypothesis $d>\delta \geqslant d^{\prime}$. Assuming for a contradiction that the claim is false, we first construct an infinite descending filtration

$$
F=F^{0} \supset F^{1} \supset F^{2} \supset F^{3} \supset \cdots
$$




\section{FAMILIES OF COHERENT ANALYTIC SHEAVES}

such that $F_{\mathbb{D}^{*}}^{n}=F_{\mathbb{D}^{*}}$ and $F_{0}^{n}$ is not $(d, \delta)$-semistable for every $n \in \mathbb{N}$, as follows: Supposing that $F^{n}$ has already been constructed, let $B^{n} \subset F_{0}^{n}$ be the maximal $(d, \delta)$-destabilizing subsheaf of $F_{0}^{n}$, let $G^{n}:=F_{0}^{n} / B^{n}$, and let $F^{n+1}$ be the kernel of the composition $F^{n} \rightarrow F_{0}^{n} \rightarrow G^{n}$. These sheaves are related through two exact sequences

$$
\begin{gathered}
0 \rightarrow B^{n} \rightarrow F_{0}^{n} \rightarrow G^{n} \rightarrow 0, \\
0 \rightarrow G^{n} \rightarrow F_{0}^{n+1} \rightarrow B^{n} \rightarrow 0 .
\end{gathered}
$$

To explain the second, we tensor over $\mathcal{O}_{\mathbb{D}}$ the exact sequence $0 \rightarrow F^{n+1} \rightarrow F^{n} \rightarrow G^{n} \rightarrow 0$ by $0 \rightarrow \mathfrak{m} \rightarrow \mathcal{O}_{\mathbb{D}} \rightarrow \mathcal{O}_{\mathbb{D}} / \mathfrak{m} \rightarrow 0$, where $\mathfrak{m}$ is the ideal sheaf of the origin in $\mathbb{D}$, to get the following commutative diagram with exact rows and columns:

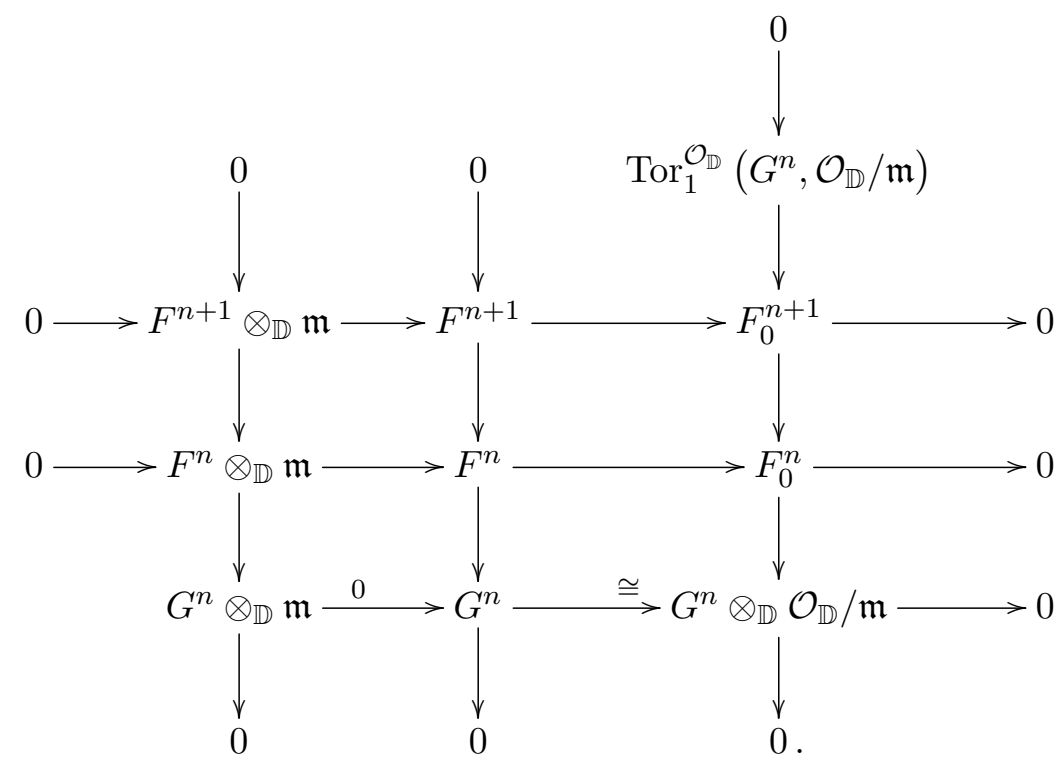

Then, using the snake lemma and the fact that $\mathfrak{m} \cong \mathcal{O}_{\mathbb{D}}$, we see that the right vertical exact sequence decomposes into two short exact sequence which are precisely the sequences (3.2) and (3.1). These exact sequences also appear in Langton's paper; cf. [MS19, Appendix C] for a detailed account of Langton's proof.

Combining the exact sequences (3.1) and (3.2), we get a self-explanatory commutative diagram with exact rows and columns:

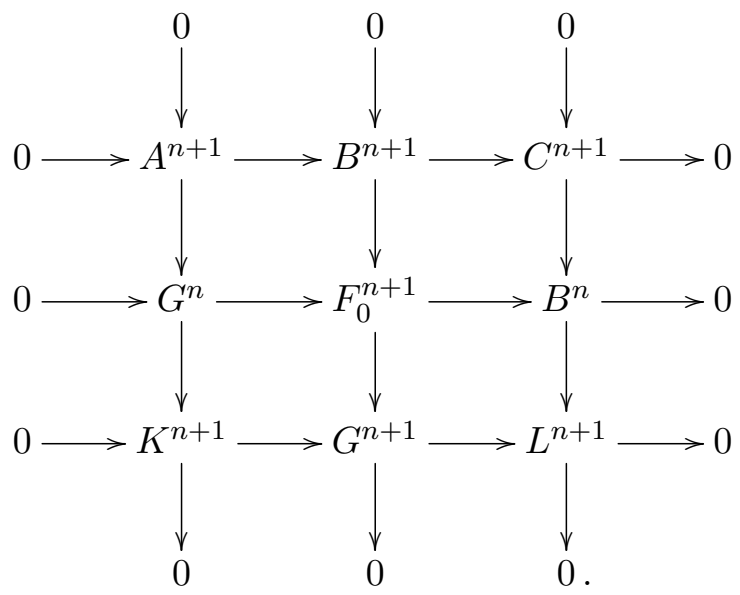


By continuity, $\operatorname{deg}_{p}\left(F_{0}^{n}\right)=\operatorname{deg}_{p}\left(F_{0}\right)$ for all $n \in \mathbb{N}$ and $p$ with $d \geqslant p \geqslant d^{\prime}$. If $C^{n+1} \neq 0$, then $C^{n+1}$ is pure $d$-dimensional as a submodule of the $(d, \delta)$-semistable module $B^{n}$ and

$$
\mu_{d, \delta}\left(B^{n+1}\right) \leqslant \mu_{d, \delta}\left(C^{n+1}\right) \leqslant \mu_{d, \delta}\left(B^{n}\right) .
$$

If $C^{n+1}=0$, we have

$$
\mu_{d, \delta}\left(B^{n+1}\right)=\mu_{d, \delta}\left(A^{n+1}\right) \leqslant \mu_{d, \delta}\left(G^{n}\right)<\mu_{d, \delta}\left(B^{n}\right)
$$

by the choice of $B^{n} \subset F_{0}^{n}$. We also have $\mu_{d, \delta}\left(B^{n}\right)>\mu_{d, \delta}\left(F_{0}\right)$; hence, the sequence $\left(\mu_{d, \delta}\left(B^{n}\right)\right)_{n}$ is descending and bounded from below. In fact, only the quotient $\operatorname{deg}_{\delta} / \operatorname{deg}_{d}$ may vary on this sequence. At this point, if we knew the degree functions to be discrete, we would conclude that the sequence $\left(\mu_{d, \delta}\left(B^{n}\right)\right)_{n}$ is eventually stationary. In our situation, we need to construct further objects in order to get the eventually stationary behaviour of this sequence.

The idea is to introduce at each formation step of the subsheaves $F^{n}$ a decomposition of $F_{0}^{n}$ into smaller and smaller building blocks as $n$ increases. At each step, we will get a collection $\mathcal{C}^{n}$ of $2^{n+1}$ building blocks. We show that this crumbling process must eventually stop, leading to the desired eventually stationary behaviour. We indicate below the first three steps of this process.

Step 0: The decomposition is given by the exact sequence $(3.1)$ for $n=0$. We set $\mathcal{C}^{0}:=$ $\left(B^{0}, G^{0}\right)$.

Step 1: The case $n=1$ for the sequences (3.1) and (3.2) leads to a diagram of type (3.4). We set $\mathcal{C}^{1}:=\left(A^{1}, C^{1}, K^{1}, L^{1}\right)$. We may view $\mathcal{C}^{1}$ as the result of cutting $\mathcal{C}^{0}$ into pieces by using (3.2). The reconstruction of $B^{0}, B^{1}, G^{0}, G^{1}, F_{0}^{0}, F_{0}^{1}$ is possible starting from $\mathcal{C}^{1}$.

Step 2: We again use (3.2), this time for $n=2$, to cut each component of $\mathcal{C}^{1}$ into two further pieces. These give the eight vertices denoted by $*$ in Figure 1 . We may count isomorphic components of $\mathcal{C}^{n}$ several times if they appear at different places in the decomposition process. Note that $B^{2}$ cuts off sub-objects of components of $\mathcal{C}^{1}$; these are represented in the vertical plane containing $B^{2}$ (the back plane).

For $n>2$, the elements of $\mathcal{C}^{n}$ will appear as vertices of the $(n+1)$-dimensional hypercube in a diagram constructed in a recursive manner as above.

Note that modulo $\mathrm{Coh}_{\delta}$, all components of $\mathcal{C}^{n}$ vanish or are $(d, \delta+1)$-semistable. Also note that for any component $E$ of $\mathcal{C}^{n}$, we have $0 \leqslant \operatorname{cycle}_{d}(E) \leqslant \operatorname{cycle}_{d}\left(F_{0}\right)$. In fact, the sum of these cycles over all components of $\mathcal{C}^{n}$ equals cycle ${ }_{d}\left(F_{0}\right)$. Thus there exists some threshold $n_{0} \in \mathbb{N}$ such that the set of $d$-cycles of components of $\mathcal{C}^{n}$ is constant for $n \geqslant n_{0}$. For $n>n_{0}$, the decomposition of components $E$ of $\mathcal{C}^{n_{0}}$ into building blocks from $\mathcal{C}^{n}$ shows that $B^{n}$ cuts off subobjects $E^{\prime}$ in such components $E$ and these sub-objects are to be used in the reconstruction of $B^{n}$ itself; in fact, they will be the quotients of a suitable filtration of $B^{n}$. If $E$ is $d$-dimensional, then such a sub-object either vanishes or is pure $d$-dimensional since $B^{n}$ is pure $d$-dimensional itself. In this second case, $\operatorname{cycle}_{d}\left(E^{\prime}\right)=\operatorname{cycle}_{d}(E)$ by our assumption on $\mathcal{C}^{n_{0}}$, and in particular $E / E^{\prime} \in \operatorname{Coh}_{\delta}(X)$ and $\operatorname{deg}_{\delta}\left(E / E^{\prime}\right)=\operatorname{deg}_{\delta}\left(\operatorname{cycle}_{\delta}\left(E / E^{\prime}\right)\right) \geqslant 0$. If $E$ is not $d$-dimensional, then $E$ has at most dimension $\delta$, and we have $\operatorname{deg}_{\delta}\left(E / E^{\prime}\right)=\operatorname{deg}_{\delta}\left(\operatorname{cycle}_{\delta}\left(E / E^{\prime}\right) \geqslant 0\right.$ in this case too. Now consider a subsequence $\left(B^{n_{k}}\right)_{k>1}$ of $\left(B^{n}\right)_{n \geqslant n_{0}}$ such that all its terms cut off non-zero sub-objects on the same sub-collection of $d$-dimensional components of $\mathcal{C}^{n_{0}}$. It follows that the sequence $\left(\operatorname{cycle}_{d}\left(B^{n_{k}}\right)\right)_{k>1}$ is constant. Moreover, using the above notation and taking sums 


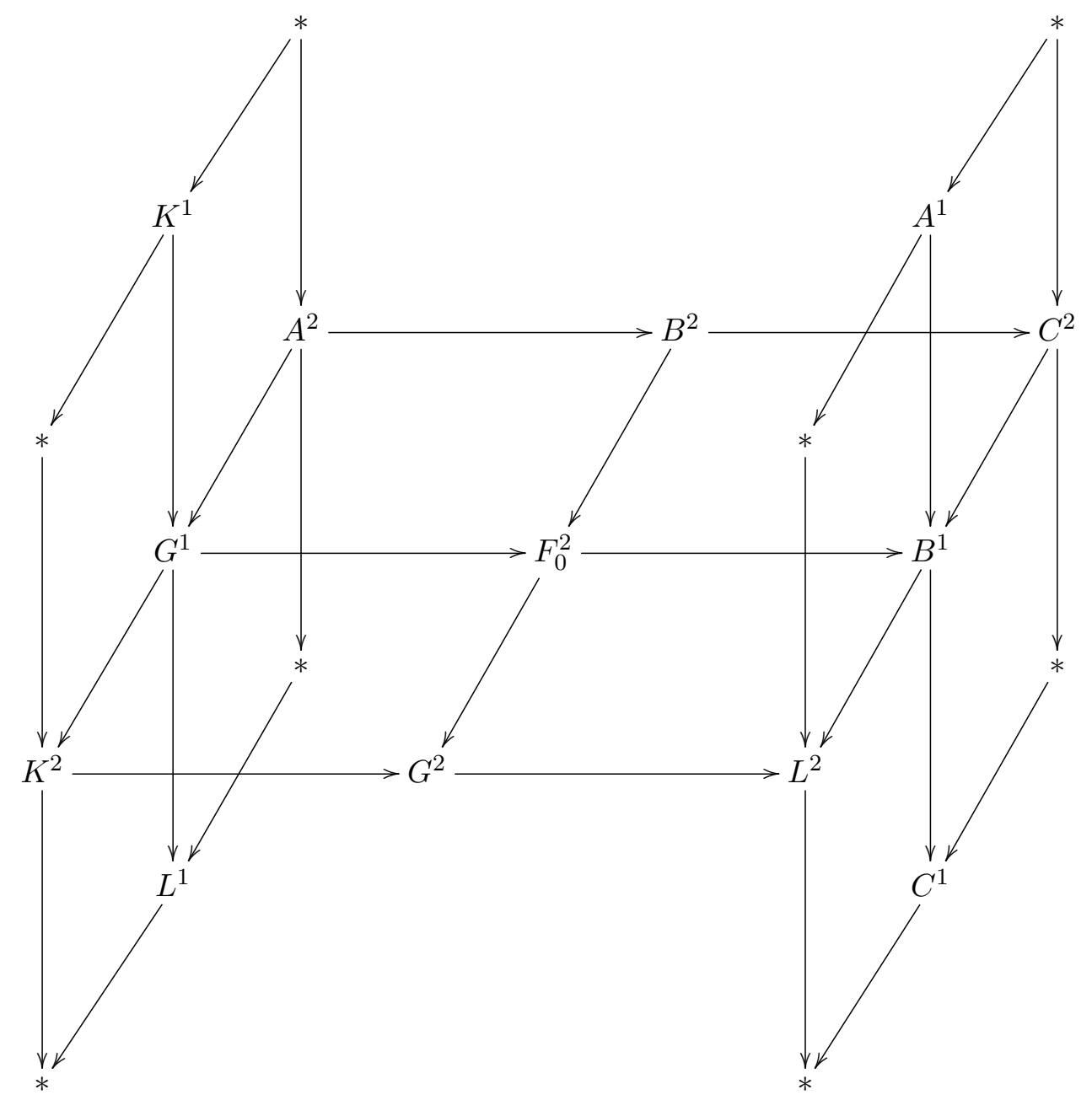

FIGURE 1. The formation of $\mathcal{C}^{2}$

over all components $E$ of $\mathcal{C}^{n_{0}}$, we find

$$
\begin{aligned}
\operatorname{deg}_{\delta}\left(B^{n_{k}}\right) & =\sum_{E} \operatorname{deg}_{\delta}\left(E^{\prime}\right)=\sum_{E} \operatorname{deg}_{\delta}(E)-\sum_{E} \operatorname{deg}_{\delta}\left(E / E^{\prime}\right) \\
& =\operatorname{deg}_{\delta}\left(F_{0}\right)-\sum_{E} \operatorname{deg}_{\delta}\left(\operatorname{cycle}_{\delta}\left(E / E^{\prime}\right)\right)=\operatorname{deg}_{\delta}\left(F_{0}\right)-\operatorname{deg}_{\delta}\left(\sum_{E} \operatorname{cycle}_{\delta}\left(E / E^{\prime}\right)\right) .
\end{aligned}
$$

It follows that the degrees in dimension $\delta$ of the cycles $\sum_{E} \operatorname{cycle}_{\delta}\left(E / E^{\prime}\right)$ are bounded, and thus by our assumption on the degree functions, they may attain only a finite number of values when $k$ varies. This implies that the sequence $\left(\mu_{d, \delta}\left(B^{n_{k}}\right)\right)_{k}$ is eventually stationary; hence, $\left(\mu_{d, \delta}\left(B^{n}\right)\right)_{n}$ is also eventually stationary.

We now continue the proof of Claim 3.2 following again [HL10]. By the above, we may assume that the sequence $\left(\mu_{d, \delta}\left(B^{n}\right)\right)_{n}$ is even constant. Then the inequalities (3.6) show that $C^{n+1} \neq 0$ for all $n$, and using (3.5) we further find $\mu_{d, \delta}\left(B^{n+1}\right)=\mu_{d, \delta}\left(C^{n+1}\right)=\mu_{d, \delta}\left(B^{n}\right)$. Thus $A^{n+1}$ either vanishes or it is $(d, \delta)$-semistable with $\mu_{d, \delta}\left(A^{n+1}\right)=\mu_{d, \delta}\left(B^{n+1}\right)=\mu_{d, \delta}\left(C^{n+1}\right)$. In the latter case, we would have $\mu_{d, \delta}\left(B^{n+1}\right)=\mu_{d, \delta}\left(A^{n+1}\right) \leqslant \mu_{d, \delta}\left(G^{n}\right)<\mu_{d, \delta}\left(B^{n}\right)$, which is impossible. 
Hence, from diagram (3.4), we get $A^{n+1}=0, B^{n+1} \cong C^{n+1} \subset B^{n}, G^{n} \cong K^{n+1} \subset G^{n+1}$ for all $n \in \mathbb{N}$. Moreover, since the sequence $\left(\operatorname{cycle}_{d}\left(B^{n}\right)\right)_{n}$ is eventually stationary, we may as well suppose that it is constant. It follows that $L^{n+1} \in \mathrm{Coh}_{\delta-1}$. In particular, the ascending sequence of pure $d$-dimensional sheaves $G^{n}$ is constant modulo $\operatorname{Coh}_{d-2}(X)$; thus their reflexive hulls $\left(G^{n}\right)^{D D}$ are all the same, and in particular the ascending sequence $\left(G^{n}\right)_{n}$ of subsheaves of $\left(G^{0}\right)^{D D}$ is eventually stationary. We again assume for simplicity that this sequence too is constant. So the central vertical and horizontal exact sequences of diagram (3.4) are split. From now on, we write $G:=G^{n}, B:=B^{n}$ and $Q^{n}:=F / F^{n}$. Via the splittings $F_{0}^{n} \cong B \oplus G$, the morphisms $F_{0}^{n+1} \rightarrow F_{0}^{n}$ from diagram (3.3) become compositions $B \oplus G \rightarrow B \rightarrow B \oplus G$ of the natural projections and injections. Hence, the cokernel of the composition $F_{0}^{n} \rightarrow F_{0}^{n-1} \rightarrow \cdots \rightarrow F_{0}^{0}$ is isomorphic to $G$. All in all, we obtain that $Q_{0}^{n} \cong G$. From the diagram

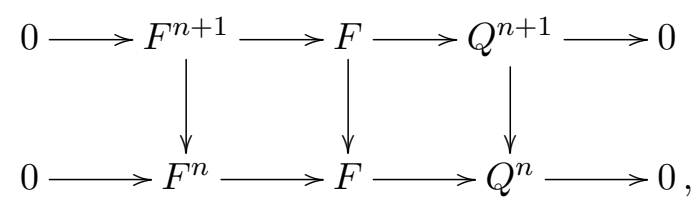

we see that $\operatorname{Ker}\left(Q^{n+1} \rightarrow Q^{n}\right) \cong \operatorname{Coker}\left(F^{n+1} \rightarrow F^{n}\right) \cong G$. Next, we will show by induction on $n$ that $Q^{n}$ is flat over $\mathcal{O}_{\mathbb{C}} / \mathfrak{m}^{n}$. The assertion is clear for $n=1$, so we assume it true for $n$ and start proving it for $n+1$. For this, we tensor the exact sequence

$$
0 \rightarrow G \rightarrow Q^{n+1} \rightarrow Q^{n} \rightarrow 0
$$

by

$$
0 \rightarrow \mathfrak{m} / \mathfrak{m}^{n+1} \rightarrow \mathcal{O}_{\mathbb{D}} / \mathfrak{m}^{n+1} \rightarrow \mathcal{O}_{\mathbb{D}} / \mathfrak{m} \rightarrow 0
$$

over $\mathcal{O}_{\mathbb{D}}$ to get

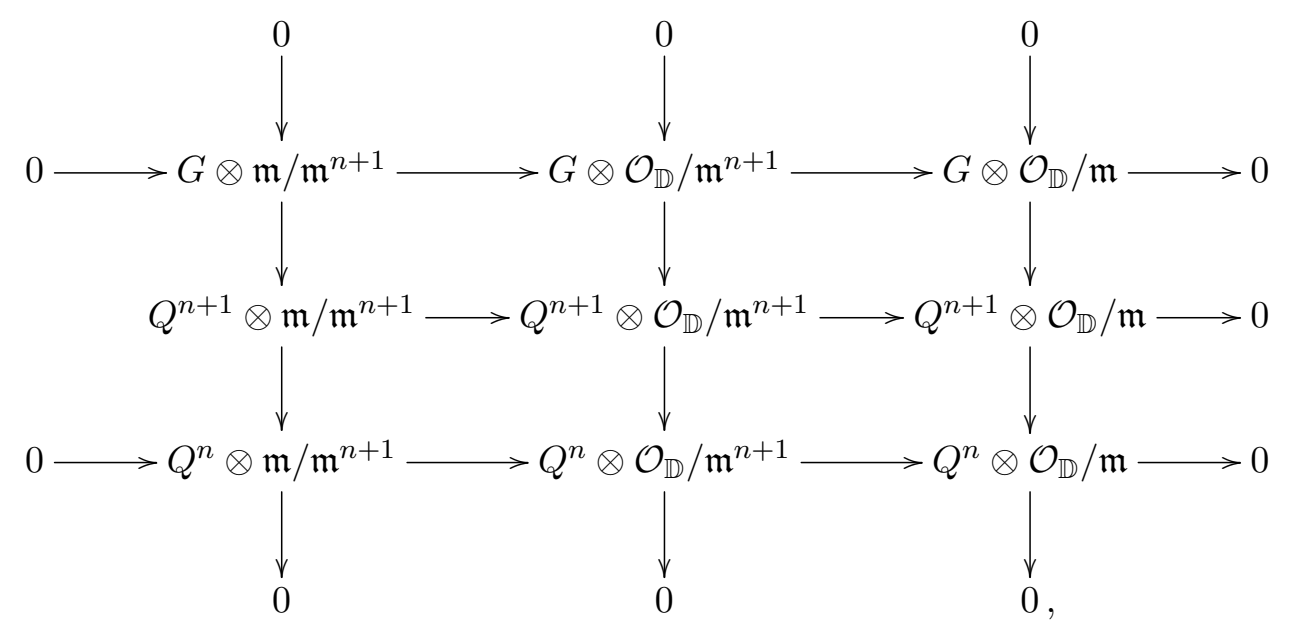

where the exactness of the first two vertical sequences follows from the induction hypothesis and from the fact that $\mathfrak{m} / \mathfrak{m}^{n+1}$ is an $\mathcal{O}_{\mathbb{C}} / \mathfrak{m}^{n}$-module. Thus the morphism $Q^{n+1} \otimes \mathfrak{m} / \mathfrak{m}^{n+1} \rightarrow$ $Q^{n+1} \otimes \mathcal{O}_{\mathbb{D}} / \mathfrak{m}^{n+1}$ is injective, and the local flatness criterion [HL10, Lemma 2.1.3] shows that $Q^{n+1}$ is a flat $\mathcal{O}_{\mathbb{D}} / \mathfrak{m}^{n+1}$-module. This gives a projective system of maps over $\mathbb{D}$ from the spaces $\operatorname{Spec}\left(\mathcal{O}_{\mathbb{D}} / \mathfrak{m}^{n}\right)$ to the relative Douady space $D_{F / X \times \mathbb{D} / \mathbb{D}}$ over $\mathbb{D}$ of quotients of $F$ which may be seen as a formal section of the natural projection of germs $\left(D_{F / X \times \mathbb{D} / \mathbb{D}}, F \rightarrow Q^{1}\right) \rightarrow(\mathbb{D}, 0)$. Such a section admits an analytic approximation to order one by Artin approximation [Art68, 


\section{FAMILIES OF COHERENT ANALYTIC SHEAVES}

Theorem 1.4.ii] showing that the projection $\left(D_{F / X \times \mathbb{D} / \mathbb{D}}, F \rightarrow Q^{1}\right) \rightarrow(\mathbb{D}, 0)$ is surjective. This contradicts the semistability of the fibres of $F$ over $\mathbb{D}^{*}$.

Proposition 3.3. Let $X$ be a compact complex manifold, and let $F$ be a $\mathbb{D}$-flat family of $d$ dimensional coherent sheaves on $X$ whose fibres over $\mathbb{D} \backslash\{0\}$ are pure of dimension $d$. Then there exists a coherent subsheaf $F^{\prime} \subset F$ coinciding with $F$ over $\mathbb{D} \backslash\{0\}$ and such that its fibre $F_{0}^{\prime}$ over 0 is also pure.

Proof. We follow the strategy of proof of Theorem 3.1, but this time we take $B_{n}:=T_{d-1}\left(F_{0}^{n}\right)$, the maximal subsheaf of $F_{0}^{n}$ of dimension at most $d-1$; cf. [HL10, Definition 1.1.4]. Then $G^{n}:=F_{0}^{n} / B^{n}$ is pure, and diagram (3.4) takes the form

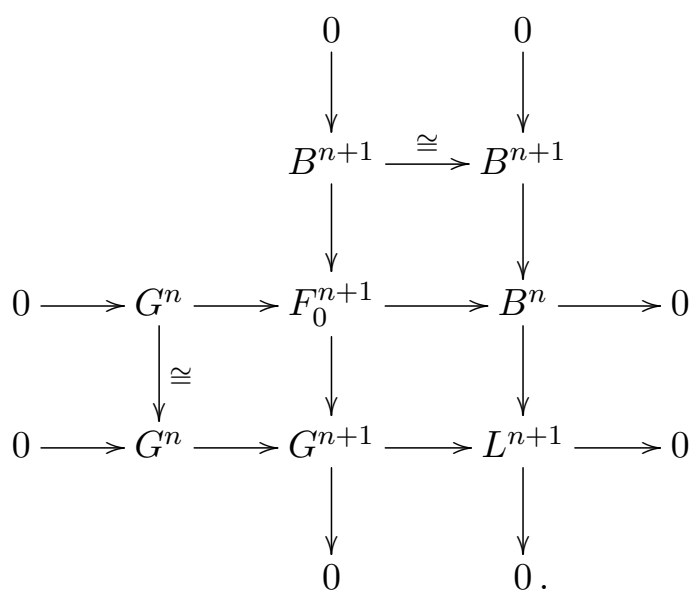

We have inequalities for associated $(d-1)$-cycles:

$$
0 \leqslant \operatorname{cycle}_{d-1}\left(B^{n+1}\right) \leqslant \operatorname{cycle}_{d-1}\left(B^{n}\right) ;
$$

hence, the sequence $\left(\text { cycle }_{d-1}\left(B^{n}\right)\right)_{n}$ must be eventually stationary, and we may assume $\operatorname{dim}\left(L^{n}\right)$ $\leqslant d-2$ for all $n$. We then immediately get that the ascending sequence of subsheaves of $\left(G_{0}\right)^{D D}$ is eventually stationary, and as before we assume that this sequence is constant and write $G:=G^{n}$ and $B:=B^{n}$. The rest of the proof follows ad litteram the proof of Theorem 3.1 but for its last sentence, where we get a contradiction to purity instead of semistability.

\section{Families of arbitrary dimension}

We now turn our attention to the case of higher-dimensional parameter spaces. As in the previous section, we give a separate purity statement. This is the content of Proposition 4.3. For the main result of the section, a stronger assumption on the degree functions will be made which will guarantee the existence of a relative maximal destabilizing subsheaf and in particular that semistability is a Zariski-open property in flat families of coherent sheaves; see Section 2. For simplicity, we only consider smooth parameter spaces. This is no significant restriction of generality since the property stated is valid after a birational base change.

THEOREM 4.1. Let $X$ be a compact complex manifold endowed with a strong $\left(d, d^{\prime}\right)$-degree system induced by a system of strictly positive $\partial \bar{\partial}$-closed differential forms, where $0 \leqslant d^{\prime} \leqslant d$. Let further $F$ be a family over $S$ of $d$-dimensional sheaves on $X$, where $S$ is a connected smooth parameter space. Suppose that general fibres of $F$ are $\left(d, d^{\prime}\right)$-semistable, and let $Z \subset S$ be the 
union of the non-flatness locus of $F$ with the closed analytic subset of $S$ parametrizing non$\left(d, d^{\prime}\right)$-semistable sheaves. Let further $K \subset S$ be a compact subset. Then there exist a proper modification $S^{\prime} \rightarrow S$ and a coherent sheaf $F^{\prime}$ on $X \times S^{\prime}$ such that $F^{\prime}$ is flat over $S^{\prime}$, all fibres of $F^{\prime}$ over $K \times{ }_{S} S^{\prime}$ are $\left(d, d^{\prime}\right)$-semistable and $F^{\prime}$ coincides with $F_{S^{\prime}}$ over $(S \backslash Z) \times_{S} S^{\prime}$.

Proof. It is clear that we only need to deal with the finitely many irreducible components of $Z$ which meet the compact set $K$. From now on, we will assume for simplicity of notation that all irreducible components of $Z$ meet $K$. The idea of the proof is on the one hand to try to reduce the dimension of the bad set $Z$ for a suitable subsheaf of $F$ constructed by a procedure similar to that which is used in the proof of Theorem 3.1; on the other hand, it will be convenient to work in the case when $Z$ is a simple normal crossings divisor and $F$ is flat over $S$. We may reduce to this situation by repeatedly blowing up $S$ at smooth centres, by Hironaka's flattening theorem [Hir75]. Since under this requirement, the dimension of $Z$ is maximal, we introduce a "badness index" $b$ in order to control the induction process in the following way: For any irreducible component $Z_{i}$ of $Z$, we say that a proper holomorphic map $\pi_{i}: Z_{i} \rightarrow B_{i}$ is $\operatorname{good}($ for $F$ ) if the restrictions of $F$ to the fibres of $\pi_{i}$ are isotrivial; that is, the fibres of these restricted families are two by two isomorphic. Then we set $b_{i}$ to be lowest possible dimension of a base $B_{i}$ of such a good map and $b$ to be the maximum among the $b_{i}$. If $Z$ is empty, we assign to $F$ a negative badness index, $b=-\infty$ say. The strategy will be the following: We start with the flat family $F$ whose non-semistable locus is a divisor with simple normal crossings $Z=\sum_{i} Z_{i}$ and which has badness index $b$. From this data, we produce a subfamily $F^{\prime} \subset F$ with $F_{S \backslash Z}^{\prime}=F_{S \backslash Z}$ and with strictly smaller bad locus. If this bad locus is empty, then $F^{\prime}$ is the family we are looking for and we stop. If not, after a suitable proper modification $S^{\prime} \rightarrow S$, the pull-back of this non-semistable locus becomes a divisor with simple normal crossings $D^{\prime}$ on $S^{\prime}$ with badness index $b^{\prime}$ for the family $F_{S^{\prime}}^{\prime} / \operatorname{Tors}_{S^{\prime}}\left(F_{S^{\prime}}^{\prime}\right)$, where $b^{\prime}<b$. We now work with the family $F_{S^{\prime}}^{\prime} / \operatorname{Tors}_{S^{\prime}}\left(F_{S^{\prime}}^{\prime}\right)$ which may be supposed in addition to be flat over $S^{\prime}$, again by Hironaka's flattening theorem [Hir75]. It is clear that this process eventually stops.

The proof of the existence of the desired subfamily $F^{\prime} \subset F$ on $S$ will follow the same path as in the one-dimensional case by descending induction on $\delta$. The corresponding claim is the following.

Claim 4.2. Let $X$ and $F$ be as in Theorem 4.1 and moreover such that $F$ is flat over $S$ and $Z$ is a divisor with simple normal crossings. Suppose that the general fibres of $F_{Z}$ are $(d, \delta+1)$ semistable for some $\delta$ with $d \geqslant \delta \geqslant d^{\prime}$. Then there exists a subsheaf $F^{\prime} \subset F$ with $F_{S \backslash Z}^{\prime}=F_{S \backslash Z}$ with $(d, \delta)$-semistable fibres over general points of $Z$ and with non-flatness locus which is nowhere dense in $Z$.

We first take a relative maximal (semi)destabilizing subsheaf $B^{0}$ of $F_{Z}$ and put $G^{0}:=F_{Z} / B^{0}$ and $F^{1}:=\operatorname{Ker}\left(F \rightarrow G^{0}\right)$. Note that $B_{s}^{0}$ is the maximal $(d, \delta)$-(semi)destabilizing subsheaf only for general points $s \in Z$. The flatness of $B^{0}$ and $G^{0}$ likewise only holds generically over $Z$. But we can work at such general points and see that the whole proof of Claim 3.2 goes through in this new relative setting. (We of course consider relative support cycles for the components of the collections $\mathcal{C}^{n}$ this time. We also use the corresponding relative statements at each moment of the proof, such as [Art68, Theorem 1.3] for instance.) In this way, we obtain a subsheaf $F^{\prime} \subset F$ with $F_{S \backslash Z}^{\prime}=F_{S \backslash Z}$ and with $(d, \delta)$-semistable fibres over general points of $Z$. At each step of the proof, the non-flatness locus of the sheaves $F^{n}$ is nowhere dense in $Z$. Indeed, assuming this to be true for $F^{n}$, we check it for $F^{n+1}$ by using an analogue of diagram (3.3) around a point of $Z$ 
where both $F^{n}$ and $G^{n}$ are flat:

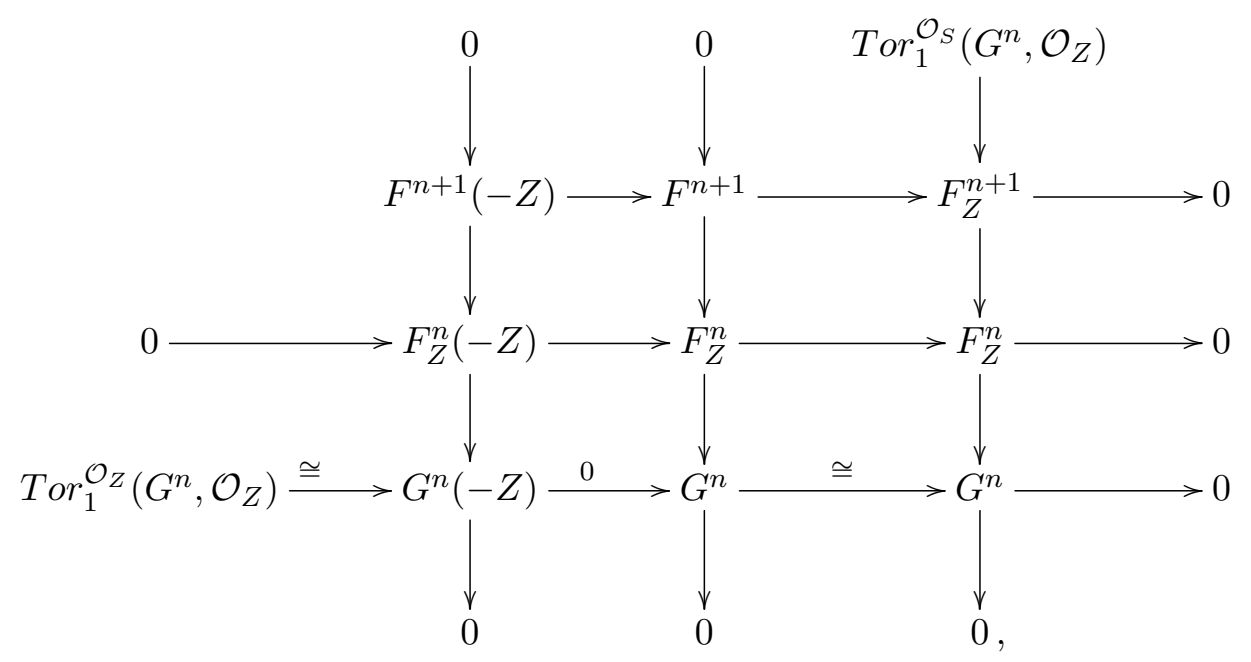

from which we immediately obtain exact sequences

$$
0 \rightarrow G^{n}(-Z) \rightarrow F_{Z}^{n+1} \rightarrow F_{Z}^{n} \rightarrow G^{n} \rightarrow 0
$$

and

$$
0 \rightarrow F^{n+1}(-Z) \rightarrow F^{n+1} \rightarrow F_{Z}^{n+1} \rightarrow 0 .
$$

From the first sequence, we infer that $F_{Z}^{n+1}$ is flat over $Z$ around the chosen point and from the second combined with [BS77, Corollaire 5.1.4] that $F^{n+1}$ is flat over $S$ around the chosen point again. This proves Claim 4.2.

If the bad locus $Z^{\prime}$ of $F^{\prime}$ on $S$ is not empty, we perform a proper modification $S^{\prime} \rightarrow S$ on $S$ so that $F^{\prime \prime}:=F_{S^{\prime}}^{\prime} / \operatorname{Tors}_{S^{\prime}}\left(F_{S^{\prime}}^{\prime}\right)$ is flat over $S^{\prime}$ with divisorial bad locus $Z^{\prime \prime}$. Let $b, b^{\prime}$ and $b^{\prime \prime}$ be the badness indices of $F, F^{\prime}$ and $F^{\prime \prime}$, respectively. Let $Z_{i} \subset Z$ be an irreducible component of $Z$ and $\pi_{i}: Z_{i} \rightarrow B_{i}$ be a good map for $F$. By the construction of $F^{\prime}$, it follows that the restrictions of the family $F^{\prime}$ to the fibres of $Z_{i}^{\prime} \rightarrow B_{i}$ are isotrivial; hence, the intersection $Z_{i} \cap Z^{\prime}$ fibres over a proper Zariski subset $B_{i}^{\prime}$ of $B_{i}$. This shows that $b^{\prime}<b$. When passing to the pair $\left(S^{\prime}, F^{\prime \prime}\right)$, it is clear that good maps for $F^{\prime \prime}$ are obtained from good maps for $F^{\prime}$ by composing with $S^{\prime} \rightarrow S$. In particular, one has $b^{\prime \prime} \leqslant b^{\prime}<b$. We have thus completed the induction argument and the proof of the theorem.

As in the case of one-dimensional bases, the previous arguments adapt to yield the following.

Proposition 4.3. Let $X$ be a compact complex manifold, and let $F$ be a family over $S$ of $d$ dimensional sheaves on $X$, where $S$ is a connected smooth parameter space. Suppose that general fibres of $F$ are pure, and let $Z \subset S$ be the union of the non-flatness locus of $F$ with the closed analytic subset of $S$ parametrizing non-pure sheaves. Let further $K \subset S$ be a compact subset. Then there exist a proper modification $S^{\prime} \rightarrow S$ and a coherent sheaf $F^{\prime}$ on $X \times S^{\prime}$ such that $F^{\prime}$ is flat over $S^{\prime}$, all fibres of $F^{\prime}$ over $K \times_{S} S^{\prime}$ are pure and $F^{\prime}$ coincides with $F_{S^{\prime}}$ over $(S \backslash Z) \times_{S} S^{\prime}$.

\section{Application to moduli spaces of semistable sheaves}

In this section, we give an application of Theorem 4.1 to the compactness of moduli spaces of semistable sheaves. For such a result to hold, one needs boundedness for the class of sheaves one 
is considering. This is typically attained by fixing the topological type or the Hilbert polynomial of these sheaves. This is the way to think of property $\mathcal{P}$ in the statement below. The moduli spaces we consider are defined according to [HL10, Definition 4.1.1] adapted to the complex analytic set-up; that is, they corepresent the functor associating with an analytic space $S$ the set of isomorphism classes of $S$-flat families of semistable sheaves with the property $\mathcal{P}$ on $X$.

Theorem 5.1. Let $X$ be a compact complex manifold endowed with a weak $\left(d, d^{\prime}\right)$-degree system, and let $\mathcal{P}$ be an open and closed property on flat families of $d$-dimensional coherent sheaves on $X$. Suppose that the corresponding semistability property satisfies the following properties:

(1) Zariski openness in flat families of coherent sheaves

(2) boundedness when restricted to the class of sheaves with the property $\mathcal{P}$

(3) the conclusion of Theorem 4.1 when restricted to the class of sheaves with the property $\mathcal{P}$

(4) the existence of a coarse moduli space $M_{\mathcal{P}}^{\text {ss }}$ for semistable sheaves with the property $\mathcal{P}$.

Then $M_{\mathcal{P}}^{\mathrm{ss}}$ is quasi-compact.

Proof. Let $K \subset S$, and let $F$ be a compact subset of a smooth complex space and a family of coherent sheaves on $X$ over $S$ giving the boundedness of the set of isomorphism classes of semistable sheaves having property $\mathcal{P}$ on $X$; cf. [Tom16, Tom19]. By restricting $S$ to a finite number of its connected components, we may suppose that all the sheaves in the corresponding family over $S$ have the property $\mathcal{P}$. Let $S^{\text {ss }} \subset S$ be the open subset which parametrizes semistable sheaves, let $D:=S \backslash S^{\mathrm{ss}}$, and let $S^{\prime} \rightarrow S$ be the proper modification given by Theorem 4.1. Then the family $F^{\prime}$ given by the conclusion of Theorem 4.1, the universal property of $M_{\mathcal{P}}^{\text {ss }}$ and the choice of $K$ and $F$ imply the existence of a surjective morphism $K \times_{S} S^{\prime} \rightarrow M_{\mathcal{P}}^{\text {ss }}$. This proves our statement.

In order to get compactness (or properness) for such a moduli space, one needs to show that $M_{\mathcal{P}}^{\text {ss }}$ is separated. This can be checked by different methods; see for instance the following two examples. For the sake of completeness, a separation result will be proved for $(n, 0)$-semistable sheaves in Section 6.

We end this section with two examples where our methods apply.

Example 5.2. Let $X$ be an $n$-dimensional complex projective manifold, $\omega$ a Kähler class on $X$, $c_{j} \in H^{2 j}(X, \mathbb{Z})$ for $1 \leqslant j \leqslant n$ and $\mathcal{P}$ the property for a coherent sheaf on $X$ of having rank two and Chern classes equal to $c_{j}$ for $1 \leqslant j \leqslant n$. Consider the semistability condition given by the $(n, 0)$-degree system induced by $\omega$ as described in Section 2 . Then $M_{\mathcal{P}}^{\text {ss }}$ is compact by Theorem 5.1. Indeed, condition (1) was proved in [Tom19], condition (2) in [GT17], condition (3) in this paper and condition (4) as well as the separatedness in [GT20]. In fact, by [GT20], the moduli space $M_{\mathcal{P}}^{\text {ss }}$ is an algebraic space over $\mathbb{C}$, so condition (3) of Theorem 5.1 may be replaced by a requirement of properness over one-dimensional bases as provided by Theorem 3.1.

Example 5.3. Let $(X, \omega)$ be a compact Kähler surface, $r \in \mathbb{Z}_{>0}, c_{1} \in \mathrm{NS}(X), c_{2} \in H^{4}(X, \mathbb{Z})$ for $1 \leqslant j \leqslant n$ and $\mathcal{P}$ the property for a coherent sheaf on $X$ to be of rank $r$ and to have Chern classes equal to $c_{1}$ and $c_{2}$. Consider the semistability condition given by the $(2,1)$-degree system induced by $\omega$, that is, the usual slope-semistability with respect to $\omega$. Suppose moreover that there exist no properly semistable sheaves with property $\mathcal{P}$; that is, all semistable sheaves with $\mathcal{P}$ are stable. This will be the case if $c_{1}$ and $r$ are relatively prime and if $\omega$ is a generic class in the Kähler cone of $X$; see [PT17, §2.2]. Then Theorem 5.1 may also be applied to this situation. 


\section{FAMILIES OF COHERENT ANALYTIC SHEAVES}

Conditions (1) and (3) are checked as in the previous example. Condition (2) is the object of an upcoming paper by Buchdahl, Teleman and the author. Finally, the moduli of stable sheaves exists as a Hausdorff open subspace of the moduli space of simple sheaves, whose existence was proved in [KO89]. For Kähler surfaces, this example generalizes the compactness result [Tom01, Theorem $4.3(\mathrm{c})$ ] by different methods.

\section{A criterion for separatedness}

Here we prove a separation result in the complex analytic set-up, which for the sake of simplicity is only formulated for $(n, 0)$-semistable sheaves. In this special case, any semistable sheaf admits a Jordan-Hölder filtration and a unique Jordan-Hölder graduation up to isomorphism; cf. [GT20, Proposition 2.8]. This property is not in general true in the case of $(n, n-1)$-semistability, which is the situation considered by Langton. This is the reason why the conclusion of his Theorem (1) $\left[\operatorname{Lan} 75, \S 3\right.$, p. 99] cannot be as sharp as ours. Recall that if a moduli space $M_{\mathcal{P}}^{\text {ss }}$ exists, semistable sheaves with isomorphic Jordan-Hölder graduations will give the same point in $M_{\mathcal{P}}^{\text {ss }}$; see [HL10, Lemma 4.1.2]. It is therefore natural to formulate a separation criterion in terms of Jordan-Hölder graduations.

Let $X$ be an $n$-dimensional compact analytic space endowed with a weak $(n, 0)$-degree system. For the induced semistability notion, the following three results are proved as in the case of Gieseker-Maruyama semistability; cf. [HL10, Proposition 1.2.7], [Ses67, Proposition 3.1] and [LeP97, §9.3], and finally [HL10, Proposition 1.5.2], respectively.

Lemma 6.1. Let $E$ and $E^{\prime}$ be semistable sheaves on $X$, and let $\phi: E \rightarrow E^{\prime}$ be a non-zero morphism of $\mathcal{O}_{X}$-modules. Then $\mu(E) \leqslant \mu\left(E^{\prime}\right)$. If equality holds, then $\operatorname{Im}(\phi)$ is semistable and $\mu(\operatorname{Im}(\phi))=\mu(E)=\mu\left(E^{\prime}\right)$. If moreover the $n$-degree of $\operatorname{Im}(\phi)$ coincides with the $n$-degree of $E$ or with the n-degree of $E^{\prime}$, then $\operatorname{Im}(\phi)$ is isomorphic to $E$ or to $E^{\prime}$, respectively.

Lemma 6.2. The full subcategory $\operatorname{Coh}^{\mathrm{ss}}\left(X ; \operatorname{deg}_{n}, \ldots, \operatorname{deg}_{0} ; \mu\right)$ of the category of coherent sheaves on $X$, whose objects are the semistable sheaves with fixed slope vector $\mu$ and the zero-sheaf, is abelian, noetherian and artinian.

Lemma 6.3 (Jordan-Hölder filtrations). Any semistable sheaf on $X$ has a Jordan-Hölder filtration in the sense of [HL10, Definition 1.5.1]. The associated graded sheaf is unique up to isomorphism.

We denote the Jordan-Hölder graduation of a semistable sheaf $E$ by $\operatorname{gr}_{\mathrm{JH}}(E)$.

We can now state the main result of this section.

THEOREM 6.4. Let $X$ be an $n$-dimensional reduced compact complex space endowed with an $(n, 0)$-degree system, and let $E$ and $F$ be two $\mathbb{D}$-flat families of semistable $n$-dimensional sheaves on $X$ which are fibrewise isomorphic over $\mathbb{D}^{*}$. Then

$$
\operatorname{gr}_{\mathrm{JH}}\left(E_{0}\right) \cong \operatorname{gr}_{\mathrm{JH}}\left(F_{0}\right) \text {. }
$$

Proof. We start by showing that after we shrink $\mathbb{D}$ around 0 if necessary, a morphism $\phi: E \rightarrow F$ exists inducing isomorphisms in all fibres over $\mathbb{D}^{*}$. For this, we denote by $p: X \times \mathbb{D} \rightarrow \mathbb{D}$ the second projection, and by $\mathcal{H}:=\mathcal{H}_{\mathrm{om}_{\mathcal{O}_{X \times \mathbb{D}}}}(p ; E, F)=p_{*} \mathcal{H} \mathrm{omO}_{X \times \mathbb{D}}(E, F)$ the relative Hom-sheaf with respect to $p$. This is a coherent sheaf on $\mathbb{D}$ of rank at least one. Let $s_{1,0}, \ldots, s_{m, 0}$ be generators of the stalk $\mathcal{H}_{0}$ as an $\mathcal{O}_{\mathbb{D}, 0}$-module. These elements have representatives $s_{1}, \ldots, s_{m} \in \mathcal{H}(U)$ over a small neighbourhood $U$ of 0 in $\mathbb{D}$ which will also generate any other stalk $\mathcal{H}_{t}$, for $t \in U$. For 
some $t \in U \backslash\{0\}$, we may thus consider a combination $\phi$ of the sections $s_{1}, \ldots, s_{m}$ over $\mathcal{O}_{\mathbb{D}}(U)$ which restricts to an isomorphism at $t$. Then $\phi$ will restrict to an isomorphism at any point in a small neighbourhood of 0 except possibly at 0 . We shrink $\mathbb{D}$ accordingly.

We denote by $\phi_{0}: E_{0} \rightarrow F_{0}$ the restriction of $\phi$ to the central fibre and by $E^{\prime}$ the kernel of the composition

$$
E \rightarrow F \rightarrow F_{0}
$$

Note that by the continuity of the degree functions, we have $\operatorname{deg}_{p}\left(E_{0}\right)=\operatorname{deg}_{p}\left(F_{0}\right)$ for all $p$. If $\phi_{0}$ is an isomorphism, the statement of Theorem 6.4 is clear. This happens if and only if $\operatorname{Coker}\left(\phi_{0}\right)=0$ if and only if $\operatorname{Coker}(\phi)=0$, as follows from the commutative diagram with exact rows and columns

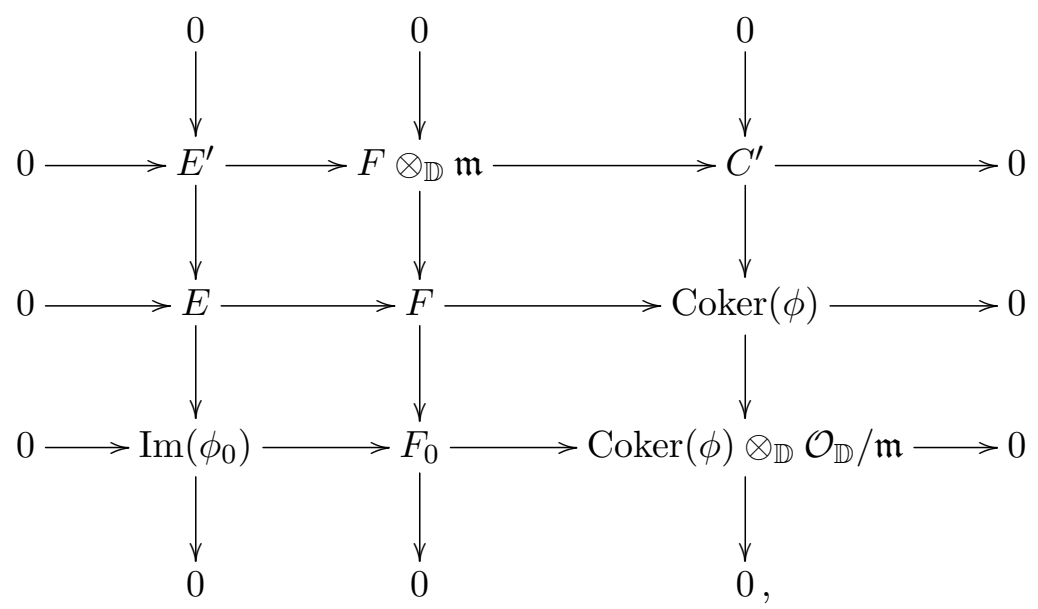

where $C^{\prime}:=\operatorname{Ker}\left(\operatorname{Coker}(\phi) \rightarrow \operatorname{Coker}(\phi) \otimes_{\mathbb{D}} \mathcal{O}_{\mathbb{D}} / \mathfrak{m}\right.$ and, as before, $\mathfrak{m}$ is the ideal sheaf of $\{0\}$ in $\mathbb{D}$. Note that the $\mathcal{O}_{X}$-module $\operatorname{Coker}\left(\phi_{0}\right) \cong \operatorname{Coker}(\phi) \otimes_{\mathbb{D}} \mathcal{O}_{\mathbb{D}} / \mathfrak{m}$ is semistable, hence pure. It thus vanishes if and only if its $n$-degree vanishes. Supposing that this is not the case, we get

$$
\operatorname{deg}_{n}\left(C^{\prime}\right)<\operatorname{deg}_{n}(\operatorname{Coker}(\phi)),
$$

where these degrees are computed for the associated $\mathcal{O}_{X}$-modules on the regular part of $X$. Note that $C:=\operatorname{Coker}(\phi)$ and $C^{\prime}$ are supported on $X \times 0:=X \times\{0\}$ and hence are $\mathcal{O}_{X \times \mathbb{D}} / p^{*}(\mathfrak{m})^{m}$ modules for $m$ high enough.

We replace our families $E$ and $F$ with $E^{\prime}$ and $F^{\prime}:=F \otimes_{\mathbb{D}} \mathfrak{m}$, respectively, and the exact sequence $0 \rightarrow E \rightarrow F \rightarrow C \rightarrow 0$ with $0 \rightarrow E^{\prime} \rightarrow F^{\prime} \rightarrow C^{\prime} \rightarrow 0$. In view of the inequality (6.1), iteration of this procedure must end after finitely many steps with an isomorphism $E^{(l)} \rightarrow F^{(l)}$, and the theorem follows if we can check that $\operatorname{gr}_{\mathrm{JH}}\left(E_{0}\right) \cong \operatorname{gr}_{\mathrm{JH}}\left(E_{0}^{\prime}\right)$ and $\operatorname{gr}_{\mathrm{JH}}\left(F_{0}\right) \cong \operatorname{gr}_{\mathrm{JH}}\left(F_{0}^{\prime}\right)$. The second equality is clear since $F_{0}^{\prime} \cong F_{0}$. It remains to establish the first one.

Tensoring the sequence $0 \rightarrow E^{\prime} \rightarrow E \rightarrow \operatorname{Im}\left(\phi_{0}\right) \rightarrow 0$ by $\mathcal{O}_{\mathbb{D}} / \mathfrak{m}$ over $\mathcal{O}_{\mathbb{D}}$, we get

$$
0 \rightarrow \mathcal{T}_{\text {or }_{1}}^{\mathbb{D}}\left(\operatorname{Im}\left(\phi_{0}\right), \mathcal{O}_{\mathbb{D}} / \mathfrak{m}\right) \rightarrow E_{0}^{\prime} \rightarrow E_{0} \rightarrow \operatorname{Im}\left(\phi_{0}\right) \rightarrow 0
$$

We also get $\mathcal{T}_{\text {or }} \mathbb{D}\left(\operatorname{Im}\left(\phi_{0}\right), \mathcal{O}_{\mathbb{D}} / \mathfrak{m}\right) \cong \operatorname{Im}\left(\phi_{0}\right) \otimes_{\mathbb{D}} \mathcal{O}_{\mathbb{D}} / \mathfrak{m}$ by tensoring the sequence $0 \rightarrow \mathfrak{m} \rightarrow \mathcal{O}_{\mathbb{D}} \rightarrow$ $\mathcal{O}_{\mathbb{D}} / \mathfrak{m} \rightarrow 0$ by $\operatorname{Im}\left(\phi_{0}\right)$. Thus $\operatorname{Ker}\left(E_{0}^{\prime} \rightarrow E_{0}\right) \cong \operatorname{Im}\left(\phi_{0}\right) \otimes_{\mathbb{D}} \mathcal{O}_{\mathbb{D}} / \mathfrak{m} \cong \operatorname{Im}\left(\phi_{0}\right)$, and we get devissages by semistable sheaves

$$
\begin{aligned}
& 0 \rightarrow \operatorname{Ker}\left(\phi_{0}\right) \rightarrow E_{0} \rightarrow \operatorname{Im}\left(\phi_{0}\right) \rightarrow 0 \\
& 0 \rightarrow \operatorname{Im}\left(\phi_{0}\right) \rightarrow E_{0}^{\prime} \rightarrow \operatorname{Ker}\left(\phi_{0}\right) \rightarrow 0
\end{aligned}
$$

for $E_{0}$ and for $E_{0}^{\prime}$ on $X$, hence $\operatorname{gr}_{\mathrm{JH}}\left(E_{0}\right) \cong \operatorname{gr}_{\mathrm{JH}}\left(E_{0}^{\prime}\right)$, and the theorem is proven. 


\section{FAMILIES OF COHERENT ANALYTIC SHEAVES}

\section{ACKNOWLEDGEMENTS}

I wish to thank Sergei Ivashkovich for pointing out to me Hirschowitz's paper [Hir80], which discusses the two definitions of meromorphic mappings.

\section{REFERENCES}

AG06 V. Ancona and B. Gaveau, Differential forms on singular varieties: de Rham and Hodge theory simplified, Pure and Applied Mathematics (Boca Raton), vol. 273 (Chapman \& Hall/CRC, Boca Raton, FL, 2006).

And09 Y. André, Slope filtrations, Confluentes Math. 1 (2009), no. 1, 1-85; doi:10.1142/ S179374420900002X.

Art68 M. Artin, On the solutions of analytic equations, Invent. Math. 5 (1968), no. 4, 277-291; doi: 10.1007/BF01389777.

Bar78 D. Barlet, Convexité de l'espace des cycles, Bull. Soc. Math. France 106 (1978), no. 4, 373-397; doi:10.24033/bsmf.1878.

BH69 T. Bloom and M. Herrera, De Rham cohomology of an analytic space, Invent. Math. 7 (1969), no. 4, 275-296; doi:10.1007/BF01425536.

BM14 D. Barlet and J. Magnússon, Cycles analytiques complexes. I. Théorèmes de préparation des cycles, Cours Spécialisés, vol. 22 (Soc. Math. France, Paris, 2014).

BS77 C. Bănică and O. Stănăşilă, Méthodes algébriques dans la théorie globale des espaces complexes (Gauthier-Villars, Paris, 1977).

CP19 F. Campana and M. Păun, Foliations with positive slopes and birational stability of orbifold cotangent bundles, Publ. Math. Inst. Hautes Études Sci. 129 (2019), no. 1, 1-49; doi:10. 1007/s10240-019-00105-w.

DP77 P. Dolbeault and J. Poly, Differential forms with subanalytic singularities; integral cohomology; residues, Several Complex Variables (Williams Coll., Williamstown, Mass., 1975), Proc. Sympos. Pure Math., Vol. XXX, Part 1 (Amer. Math. Soc., Providence, R.I., 1977), 255-261.

GKP16 D. Greb, S. Kebekus and T. Peternell, Movable curves and semistable sheaves, Int. Math. Res. Not. 2016 (2016), no. 2, 536-570; doi:10.1093/imrn/rnv126.

Gor81 R. M. Goresky, Whitney stratified chains and cochains, Trans. Amer. Math. Soc. 267 (1981), no. 1, 175-196; doi:10.2307/1998577.

GRT16a D. Greb, J. Ross and M. Toma, Moduli of vector bundles on higher-dimensional base manifolds - construction and variation, Internat. J. Math. 27 (2016), no. 6, 1650054; doi: 10.1142/S0129167X16500543.

GRT16b , Variation of Gieseker moduli spaces via quiver GIT, Geom. Topol. 20 (2016), no. 3, 1539-1610; doi:10.2140/gt.2016.20.1539.

GT17 D. Greb and M. Toma, Compact moduli spaces for slope-semistable sheaves, Algebr. Geom. 4 (2017), no. 1, 40-78; doi:10.14231/AG-2017-003.

GT20 - Moduli spaces of sheaves that are semistable with respect to a Kähler polarisation, J. Éc. polytech. Math. 7 (2020), 233-261; doi:10.5802/jep.116.

Her66 M. E. Herrera, Integration on a semianalytic set, Bull. Soc. Math. France 94 (1966), 141-180; doi:10.24033/bsmf.1637.

Hir75 H. Hironaka, Flattening theorem in complex-analytic geometry, Amer. J. Math. 97 (1975), no. 2, 503-547; doi:10.2307/2373721.

Hir80 A. Hirschowitz, Les deux types de méromorphie différent, J. reine angew. Math. 313 (1980), 157-160; doi:10.1515/crll.1980.313.157.

HL10 D. Huybrechts and M. Lehn, The geometry of moduli spaces of sheaves, 2nd ed., Cambridge Math. Lib. (Cambridge Univ. Press, Cambridge, 2010); doi:10.1017/CB09780511711985. 


\section{TOMA}

Joy07 D. Joyce, Configurations in abelian categories. III. Stability conditions and identities, Adv. Math. 215 (2007), no. 1, 153-219; doi:10.1016/j.aim.2007.04.002.

KO89 S. Kosarew and C. Okonek, Global moduli spaces and simple holomorphic bundles, Publ. Res. Inst. Math. Sci. 25 (1989), no. 1, 1-19; doi:10.2977/prims/1195173759.

Lan75 S. G. Langton, Valuative criteria for families of vector bundles on algebraic varieties, Ann. of Math. 101 (1975), no. 1, 88-110; doi:10.2307/1970987.

LeP97 J. Le Potier, Lectures on vector bundles, Cambridge Stud. Adv. Math., vol. 54 (Cambridge Univ. Press, Cambridge, 1997).

LT95 M. Lübke and A. Teleman, The Kobayashi-Hitchin correspondence (World Sci. Publ. Co., Inc., River Edge, NJ, 1995); doi:10.1142/2660.

Mar96 M. Maruyama, Construction of moduli spaces of stable sheaves via Simpson's idea, Moduli of Vector Bundles (Sanda, 1994; Kyoto, 1994), Lecture Notes in Pure and Appl. Math., vol. 179 (Dekker, New York, 1996), 147-187.

MS19 M. Maslovarić and H. Seppänen, Multi-Gieseker semistability and moduli of quiver sheaves, Beitr. Algebra Geom. 60 (2019), no. 2, 257-332; doi:10.1007/s13366-018-0417-3.

PT17 A. Perego and M. Toma, Moduli spaces of bundles over nonprojective K3 surfaces, Kyoto J. Math. 57 (2017), no. 1, 107-146; doi:10.1215/21562261-3759540.

Ses67 C.S. Seshadri, Space of unitary vector bundles on a compact Riemann surface, Ann. of Math. 85 (1967), no. 2, 303-336; doi:10.2307/1970444.

Sto58 W. Stoll, Über meromorphe Abbildungen komplexer Räume. II, Math. Ann. 136 (1958), no. 5, 393-429; doi:10.1007/BF01347792.

Tel10 A. Teleman, Instantons and curves on class VII surfaces, Ann. of Math. 172 (2010), no. 3, 1749-1804; doi:10.4007/annals.2010.172.1749.

Tom01 M. Toma, Compact moduli spaces of stable sheaves over non-algebraic surfaces, Doc. Math. 6 (2001), 11-29; https://www.emis. de/journals/DMJDMV/vol-06/02.html.

Tom16 _ Bounded sets of sheaves on Kähler manifolds, J. reine angew. Math. 710 (2016), 77-93; doi:10.1515/crelle-2013-0093.

Tom19 B Bounded sets of sheaves on Kähler manifolds. II, 2019, arXiv:1906.05853.

Var89 J. Varouchas, Kähler spaces and proper open morphisms, Math. Ann. 283 (1989), no. 1, 13-52; doi:10.1007/BF01457500.

Matei Toma Matei.Toma@univ-lorraine.fr

Matei Toma, Université de Lorraine, CNRS, IECL, F-54000 Nancy, France 